\title{
Modelagem de Micronutrientes para Mimosa scabrella na Região Metropolitana de Curitiba, Estado do Paraná
}

\author{
Saulo Jorge Téo(1), Sebastião do Amaral Machado(1), Afonso Figueiredo Filho(1) e Carlos Bruno Reissmann ${ }^{(1)}$ \\ (1) Universidade Federal do Paraná - UFPR, Av. Prefeito Lothário Meissner, 632, Jardim Botânico, CEP: 80210-170, Curitiba-PR. E-mail: \\ sauloteo@yahoo.com.br; samachado@ufpr.br; afig@ufpr.br; reissman@ufpr.br
}

\begin{abstract}
Resumo - O objetivo desta pesquisa foi modelar o conteúdo de micronutrientes da biomassa aérea de bracatinga (Mimosa scabrella Bentham). Ao todo, 25 árvores foram amostradas em diversos bracatingais da Região Metropolitana de Curitiba, no Estado do Paraná. As árvores foram derrubadas e seccionadas nos seguintes compartimentos: folhas, galhos $<4 \mathrm{~cm}$, galhos $\geq 4 \mathrm{~cm}$, madeira e casca do fuste, para determinação da massa seca e dos micronutrientes. Vários modelos matemáticos foram testados, além do procedimento stepwise. Os resultados indicaram que as equações para estimar o conteúdo de micronutrientes não apresentaram desempenho satisfatório, exceto para cobre em todos os compartimentos da biomassa e para ferro nos compartimentos da biomassa do fuste e para toda biomassa aérea. Em alguns casos, a inserção de dimensões da copa e do fuste, da idade e da altura dominante como variáveis independentes nos modelos melhorou o desempenho das equações desenvolvidas. Acredita-se que melhores resultados poderiam ter sido encontrados se a população amostrada apresentasse características mais homogêneas, como somente uma classe de sítio ou idade.
\end{abstract}

Termos para indexação: Bracatinga, conteúdo de nutrientes, ciclagem de nutrientes.

\section{Micronutrient Modeling for Mimosa scabrella Bentham in the Curitiba Metropolitan Region, Parana State, Brazil}

\begin{abstract}
The objective of this research was to model the micronutrient content for Mimosa scabrella Bentham above-ground biomass, aiming to obtain equations to estimate micronutrient content using tree dimensions as independent variables. Data came from 25 trees sampled in native Mimosa scabrella stands in the northern Curitiba metropolitan region, Parana state, Brazil. The trees were cut down and their components were separated in: leaves, branches $<4 \mathrm{~cm}$, branches $\geq 4 \mathrm{~cm}$, stem wood and stem bark. From a sample of each component it was obtained dry matter and micronutrients contents. After fitting and testing several mathematical models, it was verified that the micronutrient content equations did not present acceptable performance, except for copper in all tree components and iron in tree bole components and above-ground biomass. In some cases, using bole and crown dimensions, age and dominant height as independent variables improved the micronutrient content equations. Better results could be found if the sampled population presented homogeneous characteristics, like unique site or age class.
\end{abstract}

Index terms: Nutrient modeling, nutrient content, nutrient cycling.

\section{Introdução}

A bracatinga (Mimosa scabrella Bentham) é a espécie mais importante para a produção de lenha na região metropolitana de Curitiba. Ela ocupa uma superfície aproximada de 60.000 hectares, distribuídos por cerca de 3 mil pequenas propriedades rurais (BAGGIO; CARPANEZZI, 1997a).

O sistema de manejo tradicional da bracatinga, resultado da experiência dos pequenos proprietários rurais da região, envolve atividades que minimizam a mão-de-obra e os custos da atividade. Como algumas dessas características, é possível citar a regeneração natural induzida pela prática de queimadas, o cultivo de espécies agrícolas associadas à bracatinga no primeiro ano da rotação, a quase ausência de tratos silviculturais e atividades de manutenção do bracatingal e a simplicidade das atividades de corte, extração e carregamento da madeira do bracatingal. Todas essas características evidenciam a grande importância social, econômica e ambiental da bracatinga para a região.

Segundo Baggio e Carpanezzi (1997b), o sistema agroflorestal da bracatinga implica na exportação de nutrientes pela lenha, colheita de grãos das culturas 
agrícolas associadas e queima de resíduos pósexploração. Além disso, em muitos casos, o número de rotações em um mesmo terreno é elevado e a adubação de reposição não é aplicada, causando decréscimos de produtividade. Esses fatores tornam a nutrição e a ciclagem de nutrientes dos bracatingais um importante aspecto a ser investigado.

De acordo com Gonçalves et al. (2004), entender os processos da ciclagem de nutrientes é fundamental para o desenvolvimento de tecnologias de manejo, particularmente para determinar doses de adubação, métodos e número de aplicações.

As florestas manejadas com rotações longas e a utilização somente do fuste das árvores fazem com que a quantidade de nutrientes removidos pela exploração da floresta seja menor do que a quantidade de nutrientes no solo e as entradas de nutrientes por diferentes mecanismos (KIMMINS, 1977). No entanto, a partir da década de 1970, com a crise do petróleo, surgiram propostas de utilização dos diferentes compartimentos da biomassa da árvore, em grande escala, como fonte de energia e outros produtos.

Segundo Satoo e Madgwick (1982), a crise do petróleo da década de 1970 levou um grande número de pesquisadores a estudar a produtividade da floresta como fonte de energia e estoque de nutrientes. Esses estudos frequentemente eram relacionados com tentativas de definir o potencial de exportação de nutrientes sob diferentes sistemas de colheita florestal.

Alguns desses estudos voltaram a atenção para as relações entre a quantidade de nutrientes na biomassa das árvores com suas variáveis dendrométricas. De acordo com Van Lear et al. (1984), quando a concentração de nutrientes é multiplicada pela biomassa e estimada por meio do diâmetro, a relação é forte devido à excelente relação entre a biomassa e o diâmetro. $\mathrm{O}$ conteúdo de nutrientes pode ser estimado tanto com uma equação de regressão do conteúdo de nutrientes para um sítio específico, como pela multiplicação da biomassa média dos compartimentos da árvore pela concentração média de nutrientes.

Van Lear et al. (1984), Bellote et al. (1983), Ranger et al. (1995), Ranger e Gelhaye (2001), Laclau et al. (2000) e Hoppe (2003) desenvolveram equações para estimativa do conteúdo de nutrientes para os diferentes compartimentos da biomassa de diferentes espécies, em diferentes regiões. A maioria dessas pesquisas reportou resultados satisfatórios no ajuste de equações para estimar o conteúdo de macronutrientes. Entretanto, Bellote et al. (1983) definiram que a relação entre o conteúdo de micronutrientes nos diferentes compartimentos da biomassa de árvores de Eucalyptus grandis e suas idades não derivou em equações de boa qualidade.

O objetivo desta pesquisa foi ajustar e testar diferentes modelos matemáticos para estimativa do conteúdo de micronutrientes nos diferentes compartimentos da biomassa aérea de bracatinga.

\section{Material e Métodos}

Os dados para a realização deste estudo são provenientes de bracatingais nativos da porção norte da Região Metropolitana de Curitiba (RMC), abrangendo os municípios de Almirante Tamandaré, Campina Grande do Sul, Campo Magro e Colombo (Figura 1).

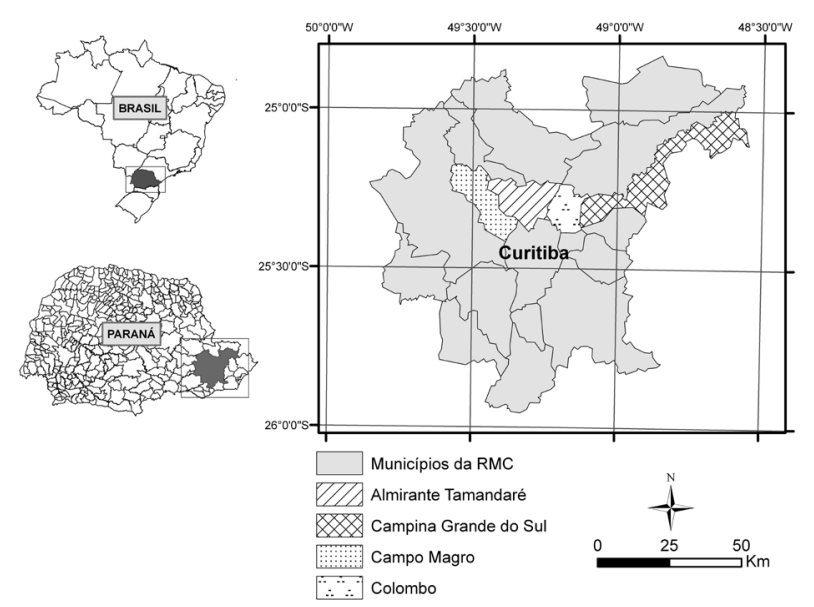

Figura 1. Mapa da Região Metropolitana de Curitiba, com destaque para a área de estudo.

A área de estudo está localizada no primeiro planalto do Paraná, cujo clima é o $\mathrm{Cfb}$ da classificação de Köeppen, com temperatura média anual de $16,5^{\circ} \mathrm{C}$ e precipitação anual em torno de $1.500 \mathrm{~mm}$. Em média, a altitude da região é de $950 \mathrm{~m}$ acima do nível do mar (MAACK, 2002).

Os solos dos bracatingais são quase invariavelmente de baixa fertilidade, classificados como Cambissolos álicos, variando de profundo a pouco profundo, principalmente em relevo forte ondulado (ZILLER et al., 1996). 
Foram amostradas 25 árvores provenientes de bracatingais nativos de diversas localidades distribuídas pela área de estudo. Os trabalhos de campo foram concentrados nos meses de junho e julho de 2007, as árvores amostradas apresentaram diâmetro médio de $11,2 \mathrm{~cm}$, variando de $7 \mathrm{~cm}$ a $20 \mathrm{~cm}$; altura total média de $14,3 \mathrm{~m}$, variando de $10,2 \mathrm{~m}$ a $19,5 \mathrm{~m}$; e idade entre 6 e 14 anos.

Os bracatingais amostrados para compor o banco de dados foram selecionados visando cobrir toda a variação da qualidade de sítios da Região Metropolitana de Curitiba, conforme a classificação de Machado et al. (1997). Em cada bracatingal, foram coletadas árvores visando cobrir diferentes classes de diâmetro, conforme inventário florestal anteriormente realizado.

No campo, as árvores foram derrubadas e seccionadas nos seguintes compartimentos da biomassa aérea: folhas, galhos menores do que $4 \mathrm{~cm}$ de diâmetro, galhos maiores ou iguais a $4 \mathrm{~cm}$ de diâmetro, madeira do fuste e casca do fuste. $\mathrm{O}$ peso verde de cada compartimento foi determinado no campo e uma amostra de cada um deles foi retirada e embalada em sacos plásticos para serem transportadas até o laboratório.

Em laboratório, todas as amostras, exceto as folhas, foram picadas e levadas à câmara de secagem a $65^{\circ} \mathrm{C}$, até peso constante para obtenção do peso seco. Posteriormente, as amostras foram moídas em moinho de facas com peneira de malha 30 mesh, e uma alíquota de aproximadamente $15 \mathrm{~g}$ foi retirada e acondicionada em frascos plásticos para realização das análises químicas dos nutrientes.

As análises químicas dos nutrientes $\mathrm{Fe}, \mathrm{Mn}, \mathrm{Cu}$ e $\mathrm{Zn}$ foram realizadas nos equipamentos do Laboratório de Biogeoquímica e Nutrição de Plantas do Departamento de Solos do Setor de Ciências Agrárias da Universidade Federal do Paraná. Os extratos da matéria seca para análise dos nutrientes foram obtidos por meio de digestão via seca e foram determinados por meio de um espectrofotômetro de absorção atômica.

Após a determinação dos pesos secos e das concentrações de nutrientes, foi possível calcular os conteúdos de $\mathrm{Fe}, \mathrm{Mn}, \mathrm{Cu}$ e $\mathrm{Zn}$ (em miligramas), que constituíram as variáveis dependentes do trabalho de modelagem. Os conteúdos de micronutrientes foram calculados para os cinco compartimentos da biomassa separadamente (folhas, galhos $<4 \mathrm{~cm}$, galhos $\geq 4 \mathrm{~cm}$, madeira e casca do fuste) e para os compartimentos da biomassa somados: folhas + galhos $<4 \mathrm{~cm}$, lenha (madeira + casca + galhos $\geq 4 \mathrm{~cm}$ ) e toda a biomassa aérea, perfazendo, ao todo, 32 variáveis dependentes.

As variáveis independentes foram: diâmetro à altura do peito (d), altura total (h), altura do fuste (hf), diâmetro de copa (dc), altura de copa (hc), razão de copa $(\mathrm{rc})$, altura dominante $\left(\mathrm{h}_{\text {dom }}\right)$ e idade; bem como, suas combinações e forma logarítmica, completando 42 variáveis independentes.

Para a medição a altura do fuste, este foi considerado até o ponto de inversão morfológica. A razão de copa (rc) foi calculada da seguinte maneira:

$$
\begin{aligned}
& \mathrm{rc}=\frac{\mathrm{hc}}{\mathrm{h}} * 100 \\
& \text { onde: } \\
& \qquad \begin{array}{l}
\mathrm{hc}=\operatorname{altura} \text { de copa }(\mathrm{m}) ; \\
\mathrm{h}=\operatorname{altura} \text { total }(\mathrm{m}) .
\end{array}
\end{aligned}
$$

Após o cálculo das variáveis dependentes e independentes para as 25 árvores amostradas, foi construída uma matriz de correlação e o procedimento stepwise foi utilizado para a seleção de variáveis na análise de regressão, utilizando-se nível de probabilidade de $10 \%$ para a entrada e saída de variáveis no modelo.

Além do procedimento stepwise, foram ajustados 15 diferentes modelos matemáticos tradicionalmente utilizados para estimativas volumétricas no meio florestal e mais 10 modelos matemáticos modificados, desenvolvidos para estimativas do conteúdo de micronutrientes nos compartimentos da biomassa da copa, e 5 modelos modificados para estimativas de micronutrientes nos compartimentos da biomassa do fuste. Esses modelos matemáticos modificados foram construídos inserindo-se dimensões da copa e do fuste com boas correlações com os conteúdos de micronutrientes como variáveis independentes.

A seleção das melhores equações foi feita com base no coeficiente de determinação ajustado $\left(\mathrm{R}_{\mathrm{aj}}^{2}\right)$, erro padrão da estimativa (syx \%) e análise gráfica dos resíduos em porcentagem. Após análise dessas estatísticas de ajuste e precisão, os modelos matemáticos foram estudados quanto à sua aceitabilidade e utilidade, ou seja, se as variáveis independentes são realmente apropriadas para oferecer as estimativas desejadas. 


\section{Resultados e Discussão}

A Tabela 1 mostra os valores médios das concentrações (mg.kg-1) e conteúdos (mg) de $\mathrm{Fe}, \mathrm{Mn}, \mathrm{Cu}$ e $\mathrm{Zn}$, nos diferentes compartimentos da biomassa aérea de bracatinga, calculadas com base nas 25 árvores amostradas. No que se refere à concentração de $\mathrm{Mn}$ e $\mathrm{Zn}$ em cada compartimento da biomassa, a seguinte ordem foi constatada: Folhas $>$ Casca $>$ Galhos $>$ Madeira. Esse resultado está de acordo com os encontrados por Baggio e Carpanezzi (1997b). Considerando todos os compartimentos da biomassa, o Mn foi o micronutriente que apresentou as maiores concentrações, seguido do Fe, $\mathrm{Cu}$ e, por último, Zn. A única exceção para essa regra foi a concentração de Fe na madeira, a qual foi maior do que a de Mn.

Tabela 1. Concentrações (mg. $\left.\mathrm{kg}^{-1}\right)$ e conteúdos médios de micronutrientes (mg) para cada um dos compartimentos da biomassa aérea de bracatinga na Região Metropolitana de Curitiba.

\begin{tabular}{ccccccc}
\hline \multirow{2}{*}{ Nutrientes } & \multicolumn{7}{c}{ Copa } & Fuste \\
\cline { 2 - 6 } & Folha & Galhos $<4^{*}$ & Galhos $\geq 4^{*}$ & Madeira & Casca & Total \\
\hline \multicolumn{7}{c}{ Concentração $\left(\mathrm{mg}^{-k^{-1}}\right)$} \\
$\mathrm{Fe}$ & 118,54 & 32,53 & 10,08 & 63,86 & 77,73 \\
$\mathrm{Mn}$ & 193,82 & 74,83 & 45,76 & 17,76 & 112,58 \\
$\mathrm{Cu}$ & 20,61 & 17,63 & 17,59 & 4,50 & 14,30 \\
$\mathrm{Zn}$ & 18,94 & 3,40 & 3,56 & 3,03 & 7,02 \\
\hline \multicolumn{7}{c}{ Conteúdo $(\mathrm{mg})$} \\
$\mathrm{Fe}$ & 279,56 & 162,85 & 58,53 & 2378,96 & 393,83 & 3264,36 \\
$\mathrm{Mn}$ & 456,99 & 382,78 & 271,94 & 661,21 & 641,13 & 2370,53 \\
$\mathrm{Cu}$ & 50,30 & 94,61 & 132,98 & 169,59 & 77,66 & 503,86 \\
$\mathrm{Zn}$ & 47,73 & 17,26 & 23,23 & 95,08 & 37,21 & 216,81 \\
\hline
\end{tabular}

* diâmetro dos galhos na ponta fina, em $\mathrm{cm}$.

Para o conteúdo total de micronutrientes na biomassa aérea de bracatinga, a seguinte ordem é verificada: $\mathrm{Fe}>$ $\mathrm{Mn}>\mathrm{Cu}>\mathrm{Zn}$. Apesar de o Mn apresentar maiores valores para a concentração na maioria dos compartimentos da biomassa, o Fe possui maior concentração na madeira, o compartimento que apresenta a maior biomassa, resultando, então, em maiores quantidades de Fe.
Na Tabela 2 são apresentadas as melhores equações ajustadas para estimativa do conteúdo de micronutrientes (mg) nos diferentes compartimentos da biomassa aérea de bracatinga, obedecendo aos critérios de seleção utilizados. Essa tabela também mostra o coeficiente de determinação ajustado $\left(\mathrm{R}^{2}{ }_{\mathrm{aj}}\right)$, erro padrão da estimativa em porcentagem (syx \%), além do fator de correção de Meyer (FM) para as equações logarítmicas. 
Tabela 2. Melhores equações desenvolvidas para estimativa do conteúdo de micronutrientes (mg) nos diferentes compartimentos da biomassa aérea de bracatinga na Região Metropolitana de Curitiba.

\begin{tabular}{|c|c|c|c|c|}
\hline $\begin{array}{c}\text { Compartimento } \\
\text { da biomassa }\end{array}$ & Equação Selecionada & $\mathbf{R}^{2}{ }_{\text {aj. }}$ & $\mathbf{s y x} \%$ & FM \\
\hline \multirow{4}{*}{ Folhas } & $\mathrm{Fe}=-1110,3+315,41 \mathrm{~d}-12,442 \mathrm{~d}^{2}-8,7608 \mathrm{dh}+0,47966 \mathrm{~d}^{2} \mathrm{~h}$ & 0,331 & 62,7 & \\
\hline & $\mathrm{Mn}=269,18+0,45965 \mathrm{dc}^{2} \mathrm{hf}^{2}-498,60 \operatorname{lndchf}^{2}$ & 0,856 & 39,6 & \\
\hline & $\mathrm{Cu}=-221,19+67,392 \mathrm{~d}-2,9946 \mathrm{~d}^{2}-2,1788 \mathrm{dh}+0,13656 \mathrm{~d}^{2} \mathrm{~h}$ & 0,825 & 29,9 & \\
\hline & $\mathrm{Zn}=-233,57+71,823 \mathrm{~d}-3,3559 \mathrm{~d}^{2}-2,3285 \mathrm{dh}+0,15296 \mathrm{~d}^{2} \mathrm{~h}$ & 0,696 & 43,7 & \\
\hline \multirow{4}{*}{ Galhos $<4 \mathrm{~cm}$} & $\operatorname{lnFe}=1,0707+0,50564 \operatorname{lnd}^{2} h$ & 0,148 & 70,8 & 1,2729 \\
\hline & $\begin{array}{l}M n=-6526,7+1559,2 d-79,179 d^{2}-106,22 d h+5,4524 d^{2} h+ \\
453,28 h\end{array}$ & 0,932 & 37,0 & \\
\hline & $\mathrm{Cu}=-282,38+93,788 \mathrm{~d}-4,4171 \mathrm{~d}^{2}-3,1435 \mathrm{dh}+0,21472 \mathrm{~d}^{2} \mathrm{~h}$ & 0,720 & 35,7 & \\
\hline & $\mathrm{Zn}=0,0070823 \mathrm{~d}^{2} \mathrm{~h}$ & 0,634 & 77,4 & \\
\hline \multirow{4}{*}{ Galhos $\geq 4 \mathrm{~cm}$} & $\operatorname{lnFe}=-3,1594+2,2625 \operatorname{lnd}+0,67800 \operatorname{lnhc}$ & 0,367 & 119,3 & 1,5045 \\
\hline & $\begin{array}{l}\mathrm{Mn}=-384,80+0,70344 \mathrm{~d}^{2} \mathrm{~h}-0,033046 \mathrm{~d}^{2} \mathrm{~h}^{2}+61,377 \mathrm{hc}-0,060713 \\
\mathrm{~d}^{2} \mathrm{hc}\end{array}$ & 0,839 & 36,4 & \\
\hline & $\mathrm{Cu}=107,40+0,54951 \mathrm{~d}^{2}-82,143 \mathrm{hc}+13,430 \mathrm{hc}^{2}-0,0068600 \mathrm{~d}^{2} \mathrm{hc}$ & 0,951 & 25,3 & \\
\hline & $\mathrm{Zn}=-2,0171+0,032375 \mathrm{~d}^{2} \mathrm{hc}$ & 0,712 & 55,3 & \\
\hline \multirow{4}{*}{ Madeira } & $\mathrm{Fe}=1551,8+25,350 \mathrm{~d}^{2}-3,8018 \mathrm{~d}^{2} \mathrm{~h}+5,0640 \mathrm{dh}^{2}-35,672 \mathrm{~h}^{2}$ & 0,861 & 25,3 & \\
\hline & $\mathrm{Mn}=216,41+0,045092 \mathrm{~d}^{2} \mathrm{hf}^{2}+76,968 \mathrm{I}-65,057 \mathrm{hdom}$ & 0,872 & 40,7 & \\
\hline & $\operatorname{lnCu}=-1,4693+2,1339 \operatorname{lnd}+0,57272 \operatorname{lnhf}$ & 0,924 & 19,9 & 1,0239 \\
\hline & $\mathrm{Zn}=0,037479 \mathrm{~d}^{2} \mathrm{~h}$ & 0,433 & 112,7 & \\
\hline \multirow{4}{*}{ Casca } & $\operatorname{lnFe}=0,85589+0,70709 \operatorname{lnd} \mathrm{d}^{2} \mathrm{hf}$ & 0,755 & 28,0 & 1,0564 \\
\hline & $M n=-1179,1+845,97 d-65,188 d^{2}-42,598 d h+3,9893 d^{2} h$ & 0,931 & 31,3 & \\
\hline & $\operatorname{lnCu}=-1,7450+0,84329 \operatorname{lnd} \mathrm{d}^{2} \mathrm{hf}$ & 0,915 & 17,4 & 1,0241 \\
\hline & $\mathrm{Zn}=9,6916+0,019338 \mathrm{~d}^{2} \mathrm{hf}$ & 0,778 & 31,6 & \\
\hline
\end{tabular}

As equações selecionadas para estimar o conteúdo de cobre e ferro apresentaram as melhores estatísticas de ajuste e precisão para os compartimentos da biomassa do fuste. Tal comportamento é parecido com a maioria das pesquisas envolvendo o ajuste de modelos matemáticos visando obter estimativas do conteúdo de nutrientes na biomassa (BELLOTE et al., 1983; RANGER et al., 1995; LACLAU et al., 2000; RANGER; GELHAYE, 2001; HOPPE, 2003).

No entanto, a qualidade dos ajustes das equações variou muito entre os diferentes micronutrientes. $\mathrm{Na}$ Tabela 2 são observados alguns valores muito baixos de $\mathrm{R}_{\text {aj. }}^{2}$ e muito altos para o syx $\%$ das equações estimativas do conteúdo de ferro e zinco. As equações selecionadas para estimativa do conteúdo de ferro mostram uma significativa melhora das estatísticas de ajuste e precisão para os compartimentos da biomassa do fuste (madeira e casca), contudo, tal melhora não ocorreu para as equações que estimam o conteúdo de zinco.

Quando os gráficos de resíduos são analisados (Figura 2 a 4), observa-se que as equações para ferro e zinco apresentam os maiores valores de resíduos para quase todos os compartimentos da biomassa, tanto é que alguns gráficos são apresentados com escalas diferentes dos demais, pois contêm alguns valores de resíduo em porcentagem altíssimos. Os gráficos em diferentes escalas são para as equações que estimam o conteúdo de zinco nos galhos $<4 \mathrm{~cm}$ e na madeira, e para ferro e zinco nos galhos $\geq 4 \mathrm{~cm}$. 

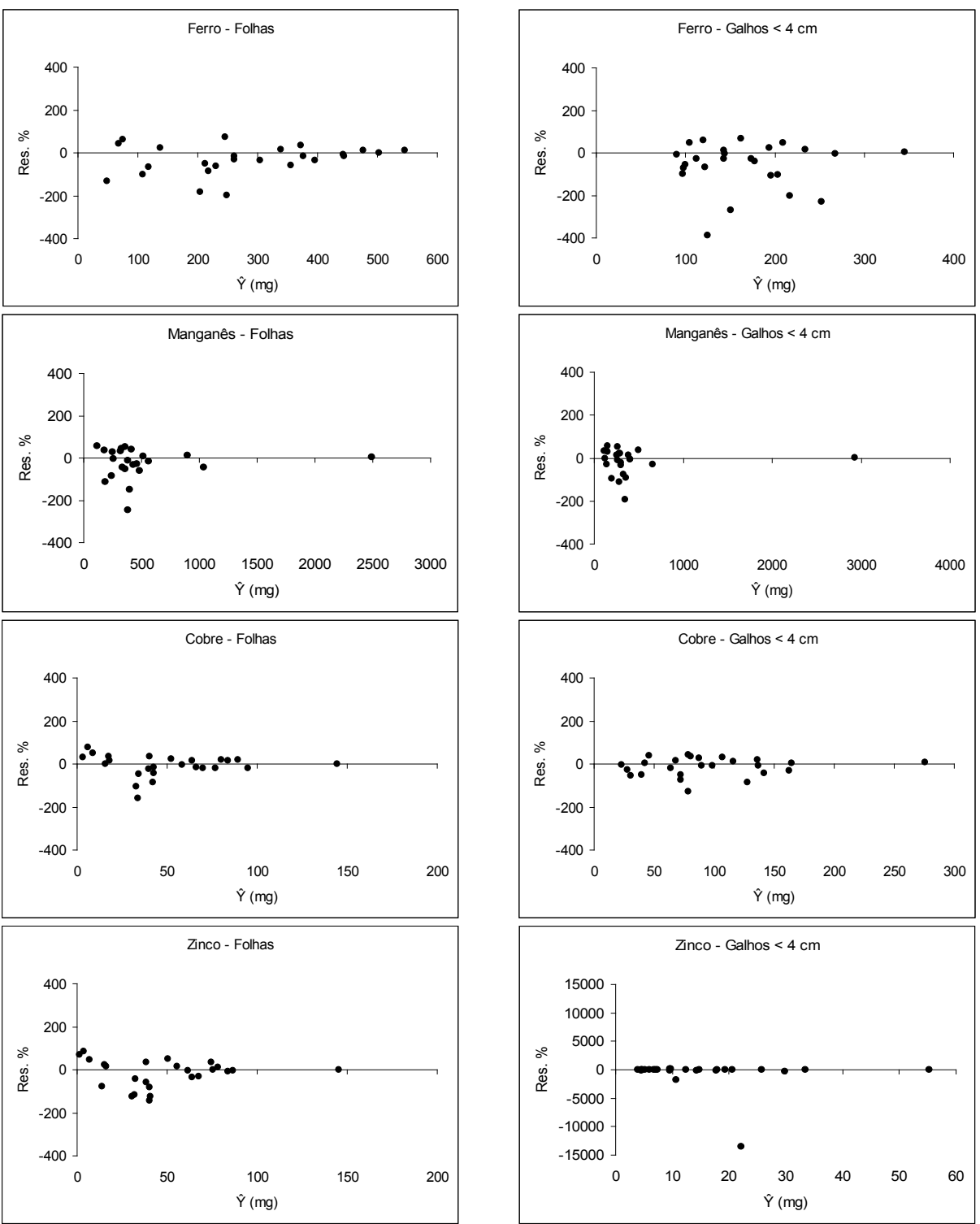

Figura 2. Distribuição dos resíduos das equações selecionadas para estimativa do conteúdo de micronuitrientes (mg) na biomassa de folhas e galhos menores que $4 \mathrm{~cm}$, para bracatinga, na Região Metropolitana de Curitiba. 

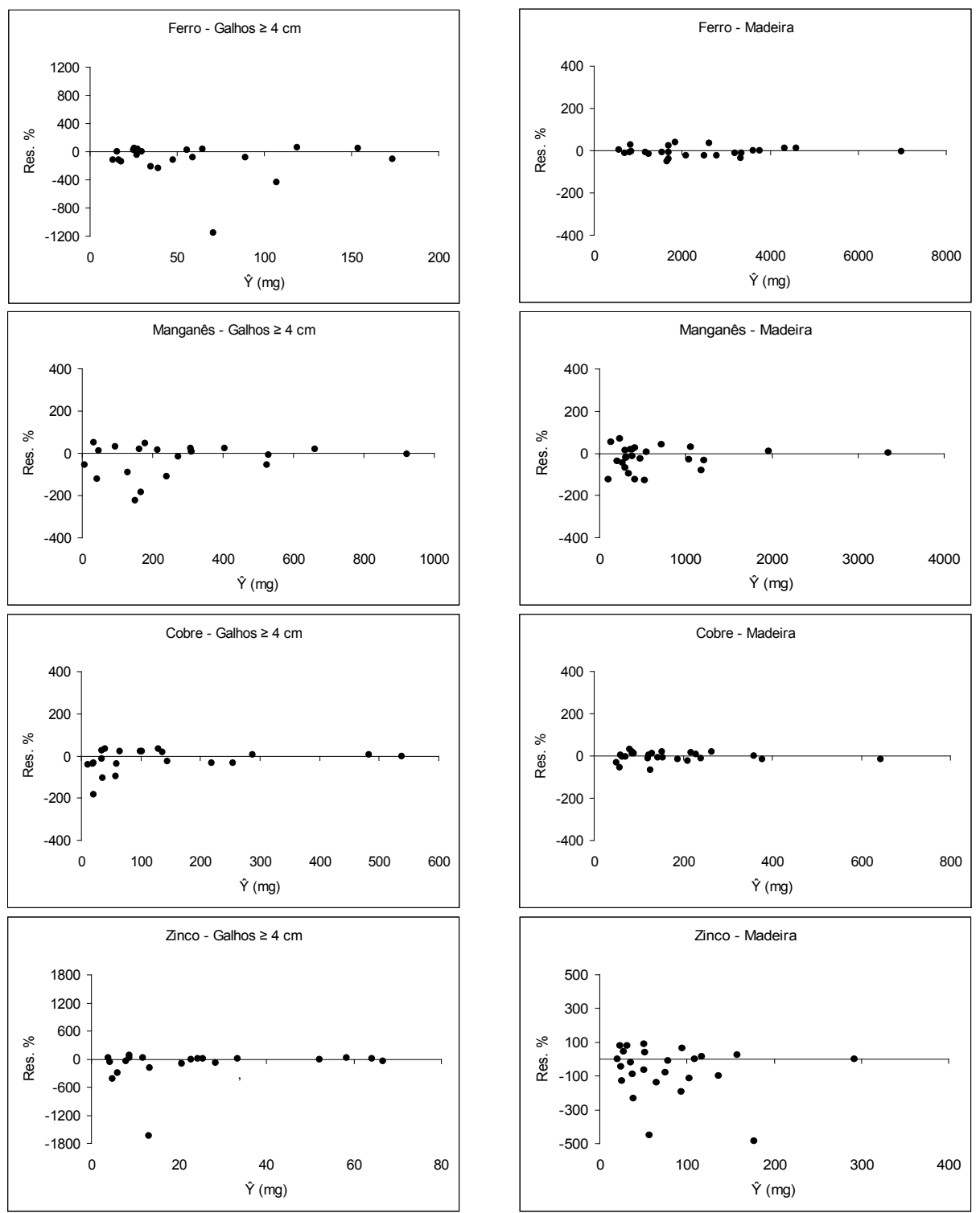

Figura 3. Distribuição dos resíduos das equações selecionadas para estimativa do conteúdo de micronuitrientes ( $\mathrm{mg}$ ) na biomassa de galhos maiores ou iguais a $4 \mathrm{~cm}$ e de madeira para bracatinga na Região Metropolitana de Curitiba. 

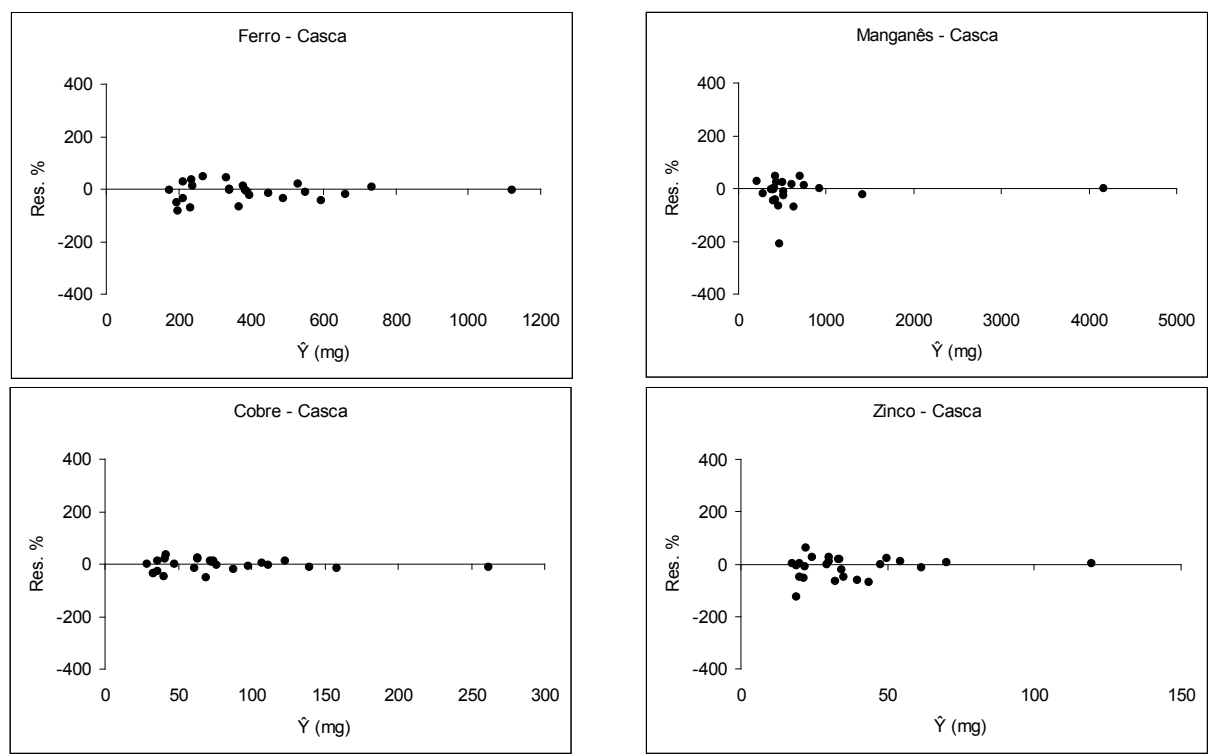

Figura 4. Distribuição dos resíduos das equações selecionadas para estimativa do conteúdo de micronuitrientes (mg) na biomassa de casca para bracatinga na Região Metropolitana de Curitiba.

Quando as distribuições de resíduo das equações estimativas de ferro e zinco foram analisadas, levantouse a hipótese de que tais valores altos de resíduo em porcentagem fossem resultado de observações atípicas (outliers). Então, como é recomendado, verificou-se a possibilidade de serem resultantes de algum erro de medição ou digitação. Sendo essa possibilidade descartada, foi procedido o ajuste dos modelos matemáticos, excetuando-se as observações que resultaram em tais valores altos de resíduos. No entanto, não foram verificadas melhoras significativas nas estatísticas de ajuste e precisão que justificassem a exclusão dessas observações do conjunto de dados.

Nos gráficos de resíduo das equações selecionadas para estimativa do conteúdo de micronutrientes, além dos altos valores de resíduos encontrados para ferro e zinco, nota-se que a distribuição de resíduos da equação que estima o conteúdo de manganês não apresenta uma distribuição de resíduos ao longo de toda a linha estimativa ( $\hat{Y}$ - conteúdo de micronutrientes estimado em miligramas). Invariavelmente, as observações se concentram nos menores valores do conteúdo de manganês e um único valor alto do conteúdo desse nutriente foi observado. Essa situação pode implicar em valores fictícios para a estatística de ajuste $\mathrm{R}^{2}{ }_{\text {aj. }}$, o qual seria mais baixo caso esse único valor alto do conteúdo de manganês não fizesse parte dos dados amostrados.
Além disso, esse único valor pode fazer com que a equação ajustada não represente a verdadeira tendência de aumento ou diminuição do conteúdo de manganês de acordo com as variáveis independentes, já que não há repetição para os valores mais altos do conteúdo desse nutriente.

As equações desenvolvidas para estimativa de cobre foram as que apresentaram as melhores estatísticas de ajuste e precisão entre os micronutrientes. As melhores distribuições de resíduo também podem ser observadas para as equações estimativas do cobre, contudo, é possível notar a presença de tendenciosidades nas distribuições de resíduo das equações nos compartimentos folhas e nos galhos $\geq 4 \mathrm{~cm}$.

Todas as equações selecionadas para estimativa do conteúdo de micronutrientes nos galhos maiores ou iguais a $4 \mathrm{~cm}$ têm como variável independente a altura de copa (hc), e a maioria das equações selecionadas para estimativa do conteúdo de micronutrientes na madeira e na casca apresentaram como variável independente a altura do fuste (hf) e sua combinação com o diâmetro à altura do peito (d) (Tabela 2).

Além das dimensões da copa e do fuste como variáveis independentes, merecem destaque as variáveis altura dominante $\left(\mathrm{h}_{\text {dom }}\right)$ e idade (I) para estimativa do conteúdo de manganês na madeira e em outros casos em que as equações stepwise não foram selecionadas como a 
equação de melhor desempenho. A seleção das variáveis idade e altura dominante pelo procedimento stepwise reforça o seu efeito significativo sobre os conteúdos de micronutrientes nos diferentes compartimentos da biomassa da bracatinga.

Observando os valores de $\mathrm{R}^{2}{ }_{\text {aj }}$, syx $\%$ e os gráficos de resíduos das equações que estimam o conteúdo de ferro para os compartimentos da biomassa da copa (folhas e galhos), é possível afirmar que a relação entre as variáveis independentes e o conteúdo de ferro é muito fraca e não pode ser generalizada para todos os bracatingais da Região Metropolitana de Curitiba, com confiabilidade. Essa mesma afirmação pode ser feita às equações para o conteúdo de zinco nos galhos e na madeira da bracatinga. Além disso, as equações que calculam o conteúdo de manganês, apesar de terem apresentado boas estatísticas de ajuste e precisão, devem ser tomadas com cautela, já que foi observada falta de uniformidade ao longo das distribuições de resíduo.

Bellote et al. (1983) não encontraram bons ajustes para equações estimativas do conteúdo de micronutrientes nos diferentes compartimentos da biomassa aérea de
Eucalyptus grandis. As equações desenvolvidas por esses autores apresentaram como variáveis independentes somente a idade das árvores. Mesmo assim, alguns resultados foram semelhantes aos encontrados nesta pesquisa.

Assim como foi observado para o conteúdo de micronutrientes em alguns compartimentos da biomassa de bracatinga, Silva (1996) e Moro et al. (2007) não encontraram boas correlações entre variáveis dendrométricas e o conteúdo de macronutrientes nos diferentes compartimentos da biomassa para Eucalyptus grandis e Pinus taeda, respectivamente.

Tendo em vista os baixos valores das estatísticas de ajuste e precisão, além de distribuição de resíduos, muitas vezes com grande amplitude de valores e com tendenciosidade para as equações apresentadas na Tabela 2, uma alternativa é mostrada na Tabela 3, que contém as equações selecionadas para estimativa do conteúdo de micronutrientes nos compartimentos da biomassa somados: folhas + galhos $<4 \mathrm{~cm}$; lenha (madeira + casca + galhos $\geq 4 \mathrm{~cm}$ ); e para toda biomassa aérea de bracatinga.

Tabela 3. Melhores equações desenvolvidas para estimativa do conteúdo de micronutrientes (mg) para os compartimentos Folhas + Galhos $<4$ cm, lenha e para a biomassa aérea de bracatinga na Região Metropolitana de Curitiba.

\begin{tabular}{|c|c|c|c|c|}
\hline $\begin{array}{c}\text { Compartimento da } \\
\text { biomassa }\end{array}$ & Equação Selecionada & $\mathbf{R}^{2}{ }_{\mathrm{aj} .}$ & $\mathbf{s y x} \%$ & FM \\
\hline \multirow{4}{*}{ Folhas + Galhos $<4 \mathrm{~cm}$} & $\mathrm{Fe}=-340,64+1691,9 \operatorname{lnd}-461,40 \operatorname{lnd}{ }^{2} \mathrm{hf}$ & 0,517 & 45,3 & \\
\hline & $\mathrm{Mn}=-8042,2+2303,1 \mathrm{~d}-127,20 \mathrm{~d}^{2}-145,22 \mathrm{dh}+8,3975 \mathrm{~d}^{2} \mathrm{~h}+489,95 \mathrm{~h}$ & 0,959 & 24,2 & \\
\hline & $\mathrm{Cu}=-503,57+161,18 \mathrm{~d}-7,4116 \mathrm{~d}^{2}-5,3223 \mathrm{dh}+0,35128 \mathrm{~d}^{2} \mathrm{~h}$ & 0,834 & 26,9 & \\
\hline & $\mathrm{Zn}=-255,96+83,155 \mathrm{~d}-3,9410 \mathrm{~d}^{2}-2,8112 \mathrm{dh}+0,18759 \mathrm{~d}^{2} \mathrm{~h}$ & 0,733 & 37,6 & \\
\hline \multirow{4}{*}{ Lenha } & $\mathrm{Fe}=\mathrm{h}\left(-168,15+37,406 \mathrm{~d}-0,47454 \mathrm{~d}^{2}\right)$ & 0,872 & 23,3 & \\
\hline & $\mathrm{Mn}=6620,6+0,11915 \mathrm{~d}^{2} \mathrm{hf}^{2}-1151,1 \operatorname{lndhf}^{2}+116,56 \mathrm{I}$ & 0,936 & 25,2 & \\
\hline & $\operatorname{lnCu}=9,3498-0,25277 \operatorname{lnd}-32,852 \mathrm{~d}^{-1}$ & 0,902 & 23,5 & 1,0220 \\
\hline & $\operatorname{lnZn}=-1,2721+0,84514 \operatorname{lnd}^{2} \mathrm{hf}$ & 0,358 & 67,6 & 1,2620 \\
\hline \multirow{4}{*}{ Total } & $\mathrm{Fe}=-7402,9+2012,5 \mathrm{~d}-100,83 \mathrm{~d}^{2}-42,633 \mathrm{dh}+4,2652 \mathrm{~d}^{2} \mathrm{~h}$ & 0,875 & 22,0 & \multirow{4}{*}{1,0172} \\
\hline & $\mathrm{Mn}=17610,2+0,22425 \mathrm{~d}^{2} h f^{2}-2739,7 \operatorname{lndhf^{2}}+142,10 \operatorname{lnhf}$ & 0,950 & 23,3 & \\
\hline & $\operatorname{lnCu}=10,604-0,56252 \operatorname{lnd}-34,298 \mathrm{~d}^{-1}$ & 0,928 & 18,7 & \\
\hline & $\mathrm{Zn}=-666,89+229,87 \mathrm{~d}-13,004 \mathrm{~d}^{2}-7,1992 \mathrm{dh}+0,60934 \mathrm{~d}^{2} h$ & 0,566 & 47,4 & \\
\hline
\end{tabular}


As equações apresentadas na Tabela 3, geralmente, apresentaram melhores desempenhos do que aquelas da Tabela 2 para os compartimentos da biomassa aérea separadamente.

As Figuras 5 e 6 mostram os resíduos das equações selecionadas para estimativa do conteúdo de micronutrientes dos compartimentos folhas + galhos $<4 \mathrm{~cm}$, lenha e biomassa aérea. Verifica-se uma melhora na distribuição dos resíduos das equações da Tabela 3 com relação às apresentadas anteriormente, na Tabela 2. Convém ressaltar que os gráficos mostrados nas Figuras 5 e 6 estão em escalas diferentes daqueles apresentados nas Figuras 2, 3 e 4 devido à menor amplitude de valores dos resíduos.
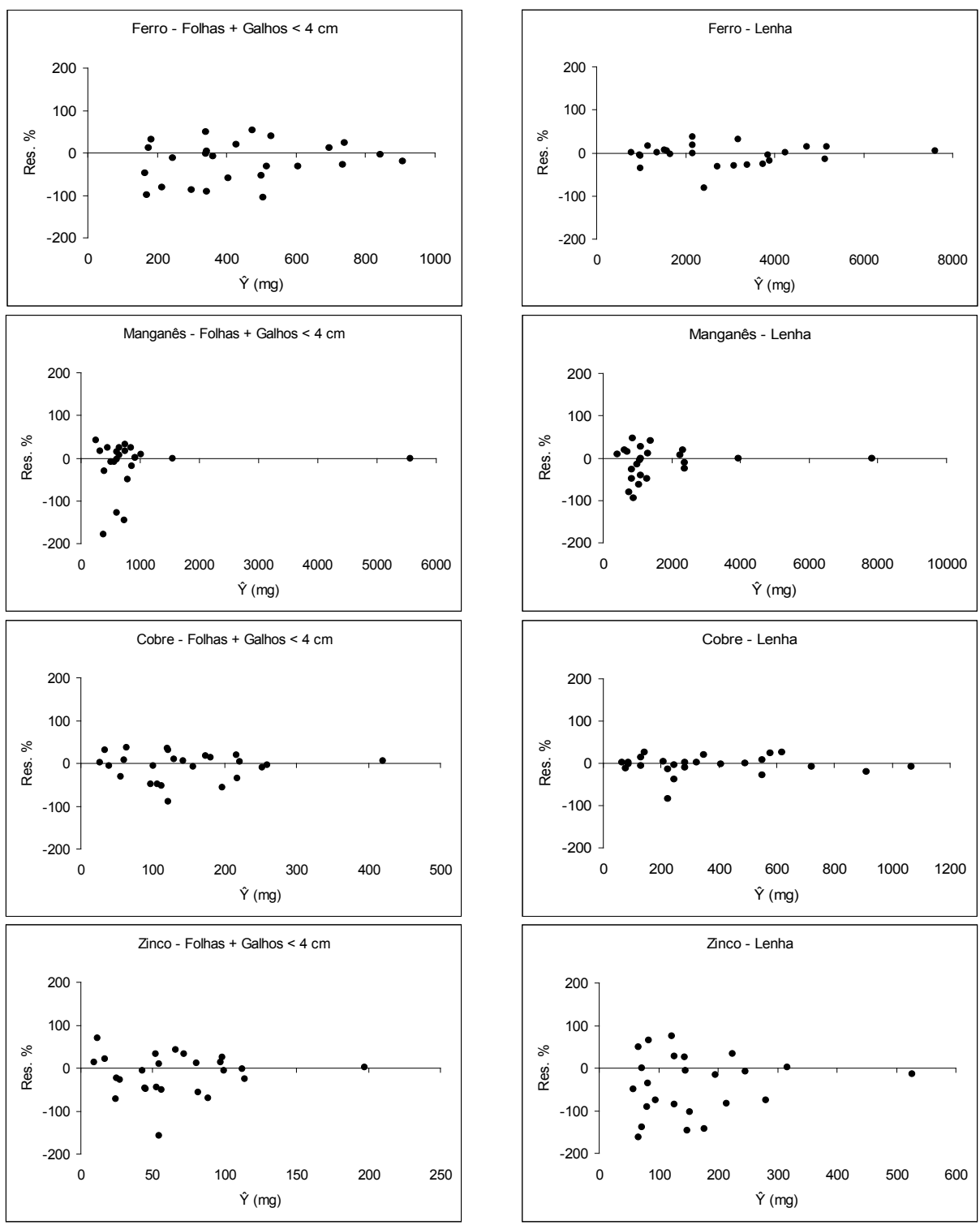

Figura 5. Distribuição dos resíduos das equações selecionadas para estimativa do conteúdo de micronuitrientes (mg) na biomassa de folhas + galhos menores que $4 \mathrm{~cm}$ e lenha de bracatinga na Região Metropolitana de Curitiba. 

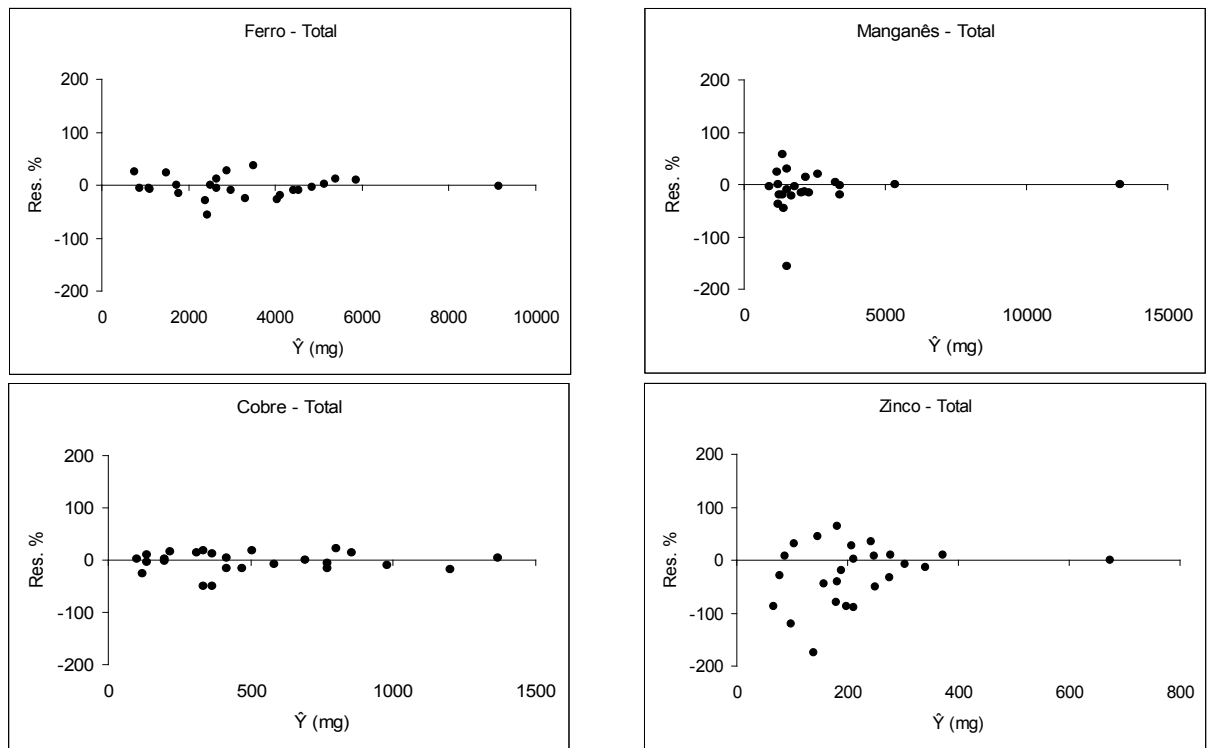

Figura 6. Distribuiçâo dos resíduos das equações selecionadas para estimativa do conteúdo de micronuitrientes ( $\mathrm{mg}$ ) na biomassa aérea de bracatinga na Região Metropolitana de Curitiba.

Apesar das melhoras na qualidade dos ajustes, e principalmente nos gráficos de resíduo, as equações selecionadas para estimativa de ferro na biomassa de folhas + galhos $<4 \mathrm{~cm}$ e de zinco para todos os compartimentos da biomassa, inclusive para toda a biomassa aérea, não apresentaram bons valores para as estatísticas de ajuste e precisão (Tabela 3). Nos gráficos de resíduos para essas equações, geralmente, observamse tendências de superestimativas, principalmente para os menores valores de $\hat{Y}$.

Desta vez, não foram observados resíduos com valores exorbitantes para as equações estimativas dos conteúdos de ferro e zinco nos compartimentos da biomassa da copa. Sendo assim, todos os gráficos puderam ser apresentados nas mesmas escalas, favorecendo a comparação das distribuições de resíduo para as equações estimativas dos diferentes micronutrientes.

Da mesma forma que para as equações da Tabela 2, para os compartimentos da biomassa separadamente, as equações para o conteúdo de manganês apresentaram bons valores para as estatísticas de ajuste e precisão, contudo, observa-se o mesmo padrão de distribuição dos resíduos, ou seja, os resíduos se concentram para os menores valores de $\hat{Y}$ e somente um valor alto do conteúdo de manganês é observado.

Por outro lado, as equações para o conteúdo de cobre, para todos os compartimentos da biomassa, e de ferro, para a biomassa de lenha e para toda a biomassa aérea, apresentaram valores de $\mathrm{R}^{2}$ aj. altos e syx \% relativamente baixos. Além disso, as distribuições de resíduo apresentaram amplitude de valores baixa, uniformidade e ausência de tendenciosidades ao longo da linha estimativa.

Para os compartimentos da biomassa somados, a grande maioria das equações stepwise tinha como variáveis independentes dimensões da copa e do fuste, como diâmetro de copa (dc), altura de copa (hc) e altura do fuste (hf). Entretanto, a variável idade (I) apareceu com menor frequência e a altura dominante $\left(\mathrm{h}_{\text {dom }}\right)$ não figurou em nenhuma das equações resultantes do procedimento stepwise.

\section{Conclusões}

Somente as equações que estimam o conteúdo de cobre e ferro para alguns compartimentos da biomassa de bracatinga apresentaram bons desempenhos e podem ser utilizadas com segurança nos bracatingais da Região Metropolitana de Curitiba, desde que seja respeitada a amplitude de variação dos dados amostrados nesta pesquisa.

As equações que estimam o conteúdo de cobre apresentaram melhores desempenhos para os compartimentos da biomassa somados, portanto, devem ser preferidas. 
Em alguns casos, a inserção de dimensões da copa e do fuste, da idade e da altura dominante melhorou os desempenhos das equações que estimam o conteúdo de micronutrientes.

Melhores resultados poderiam ter sido alcançados se os dados utilizados nesta pesquisa proviessem de uma população com características mais homogêneas, como por exemplo, somente uma classe de sítio e idade.

\section{Referências}

BAGGIO, A. J.; CARPANEZZI, A. A. Estoque de nutrientes nos resíduos da exploração de bracatingais. Boletim de Pesquisa Florestal, Colombo, n. 34, p. 17-29, 1997a.

BAGGIO, A. J.; CARPANEZZI, A. A. Exportação de nutrientes na exploração de bracatingais. Boletim de Pesquisa Florestal, Colombo, n. 34, p. 3-15. 1997 b.

BELLOTE, A. F. J.; SARRUGE, J. R.; HAAG, H. P. OLIVEIRA, G. D. de. Absorção de macronutrientes e micronutrientes pelo Eucalyptus grandis (Hill, ex-Maiden) em função da idade. Silvicultura, São Paulo, n. 32, p. 633-643, 1983.

GONÇALVES, J. L. de M.; STAPE, J. L.; BENEDETTI, V.; FESSEL, V. A. G.; GAVA, J. L. An evaluation of minimum and intensive soil preparation regarding fertility and tree nutrition. In: GONÇALVES, J. L. de M.; BENEDETTI, V. Forest nutrition and fertilization. Piracicaba: IPEF, 2004. p. 13-64.

HOPPE, J. M. Biomassa e nutrientes em Platanus $x$ acerifolia (Aiton) Willd. estabelecido no município de Dom Feliciano RS. 143 f. Tese (Doutorado em Engenharia Florestal) - Setor de Ciências Agrárias, Universidade Federal de Santa Maria, Santa Maria, 2003.

KIMMINS, J. P. Evaluation of the consequences for future tree productivity of the loss of nutriens in whole-tree harvesting. Forest Ecology and Management, Amsterdam, v. 1, n. 2, p. 169183, 1977.

LACLAU, J. P.; BOUILLET, J. P.; RANGER, J. Dynamics of biomass and nutrient accumulation in a clonal plantation of Eucalyptus in Congo. Forest Ecology and Management, Amsterdam, v. 128, n. 3, p. 181-196, 2000.
MAACK, R. Geografia física do estado do Paraná. 3. ed. Curitiba: Imprensa Oficial do Paraná, 2002. 440p.

MACHADO, S. A.; OLIVEIRA, E. B.; CARPANEZZI, A. A.; BARTOSZECK, A. C. P. S. Classificação de sítio para bracatingais na região metropolitana de Curitiba. Boletim de Pesquisa Florestal, Colombo, n. 35, p. 21-37, 1997.

MORO, L.; REISSMANN, C. B.; SILVA, H. D. da; FERREIRA, C. A.; LAVORANTI, O. J. Modelos matemáticos para estimativa de exportação de nutrientes em povoamentos de Pinus taeda L. Floresta, Curitiba, v. 37, n. 2, p. 223-230, 2007.

RANGER, J.; GELHAYE, D. Belowground biomass and nutrient content in a 47-year-old Douglas-fir plantation. Annals of Forest Science, Paris, v. 58, n. 4, p. 423-430, 2001.

RANGER, J.; MARQUES, R.; COLIN-BELGRAND, M.; FLAMMANG, N.; GELHAYE, D. The dynamics of biomass and nutrient accumulation in a Douglas-fir (Pseudotsuga menziesii Franco) stand studied using a chronosequence approach. Forest Ecology and Management, Amsterdam, v. 72, n. 2, p. 167-183, 1995.

SATOO, T.; MADGWICK, H. A. I. Forest biomass. Dordrecht: Martinus Nijhoff, 1982. 152p.

SILVA, H. D. da. Modelos Matemáticos para Estimativa da Biomassa e do Conteúdo de Nutrientes em Plantações de Eucalyptus gradis Hill (ex-Maiden) em Diferentes Idades. 101 f. Tese (Doutorado em Engenharia Florestal) - Setor de Ciências Agrárias, Universidade Federal do Paraná, Curitiba, 1996.

VAN LEAR, D. H.; WAIDE, J. B.; TEUKE, M. J. Biomass and nutrient content of a 41-year-old Lobloly pine (Pinus taeda L.) plantation on a poor site in South Carolina. Forest Science, Bethesda, v. 30, n. 2, p. 395-404, 1984.

ZILLER, S. R.; RIBASKI, J.; NEVES, E. J. M.; WISNIEWSKI, C.; CURCIO, G. R.; RACHWAL, M. F. G.; MARTINS, E. G.; MARTINEZ, C. M.; SOUZA, S. L. DE; PANZERI, C. G.; MASCHIO, W. Relação entre serapilheira acumulada, características fitossociológicas e pedológicas em quatro capoeiras de bracatinga (Mimosa scabrella Bentham) na região metropolitana de Curitiba - PR. Arquivos de Biologia e Tecnologia, Curitiba, v. 39, n. 4, p. 911-921, 1996.

Recebido em 11 de maio de 2009 e aprovado em 09 de dezembro de 2009 